\title{
Mast cell dipeptidyl peptidase I mediates survival from sepsis
}

\author{
Jon Mallen-St. Clair, ${ }^{1}$ Christine T.N. Pham, ${ }^{2}$ S. Armando Villalta, ${ }^{1}$ George H. Caughey, ${ }^{1}$ \\ and Paul J. Wolters ${ }^{1}$ \\ ${ }^{1}$ Department of Medicine and The Cardiovascular Research Institute, University of California, San Francisco, California, USA \\ ${ }^{2}$ Department of Internal Medicine and Department of Pathology and Immunology, Washington University \\ School of Medicine, St. Louis, Missouri, USA
}

\begin{abstract}
Sepsis is a common, life-threatening disease for which there is little treatment. The cysteine protease dipeptidyl peptidase I (DPPI) activates granule-associated serine proteases, several of which play important roles in host responses to bacterial infection. To examine DPPI's role in sepsis, we compared $\mathrm{DPPI}^{-/-}$and $\mathrm{DPPI}^{+/+}$mice using the cecal ligation and puncture (CLP) model of septic peritonitis, finding that $\mathrm{DPPI}^{-/-}$mice are far more likely to survive sepsis. Outcomes of CLP in mice lacking mast cell DPPI reveal that the absence of DPPI in mast cells, rather than in other cell types, is responsible for the survival advantage. Among several cytokines surveyed in peritoneal fluid and serum, IL-6 is highly and differentially expressed in $\mathrm{DPPI}^{-/-}$mice compared with $\mathrm{DPPI}^{+/+}$mice. Remarkably, deleting IL-6 expression in $\mathrm{DPPI}^{-/-}$mice eliminates the survival advantage. The increase in IL-6 in septic $\mathrm{DPPI}^{-/-}$mice, which appears to protect these mice from death, may be related to reduced DPPI-mediated activation of mast cell tryptase and other peptidases, which we show cleave IL-6 in vitro. These results indicate that mast cell DPPI harms the septic host and that DPPI is a novel potential therapeutic target for treatment of sepsis.
\end{abstract}

J. Clin. Invest. 113:628-634 (2004). doi:10.1172/JCI200419062.

\section{Introduction}

Sepsis syndrome is the consequence of the host's response to overwhelming bacterial infection and is characterized by hypotension, decreased organ perfusion, microvascular thrombosis, and organ ischemia, which lead to organ dysfunction and death (1). Innate immune cells recognize the presence of invading bacteria and initiate the host response by releasing cytokines that recruit inflammatory cells, enhance bacterial clearance, and activate adaptive immune cells to generate pathogen-specific antibodies (2). When cytokines and inflammatory cells are present in judicious amounts, the pathogens are removed without adverse consequence. Excessive amounts or imbalances of pro- and anti-inflammatory cytokines lead to sepsis syndrome and death (1).

Mast cells play a central role in protecting a host infected with bacteria $(3,4)$. Following infection, bacteria activate mast cells directly via binding of pathogen-associated molecules (e.g., LPS and peptidoglycans) (2) to pattern recognition receptors (e.g., toll-

Received for publication May 29, 2003, and accepted in revised form December 2, 2003.

Address correspondence to: Paul J. Wolters, University of California, San Francisco, Box 0911, San Francisco, California 94143-0911, USA. Phone: (415) 514-2601; Fax: (415) 476-9749; E-mail: pjwolt@itsa.ucsf.edu.

Conflict of interest: The authors have declared that no conflict of interest exists.

Nonstandard abbreviations used: dipeptidyl peptidase I (DPPI); cecal ligation and puncture (CLP); bone marrow mast cell (BMMC); mouse mast cell protease (mMCP); peritoneal mast cell (PMC). like receptors or $\mathrm{CD} 48)(5,6)$ or indirectly via binding of activated complement components to CD21/CD35 (7). Upon activation, these receptors trigger mast cell release of TNF- $\alpha$, which aids in recruiting neutrophils to the site of infection (3). Recruited neutrophils then use the serine peptidase neutrophil elastase to kill bacteria by cleaving cell wall proteins (8) or destroying virulence factors (9).

Dipeptidyl peptidase I (DPPI) is a cysteine-class hydrolase that removes $\mathrm{NH}_{2}$-terminal dipeptides from a variety of targets (10). Studies in $D P P I^{-/-}$mice show that DPPI is required for propeptide removal and activation of the granule-associated serine peptidases granzyme A, granzyme $B$, neutrophil elastase, cathepsin $\mathrm{G}$, and mast cell chymases, all of which are inactive in $\mathrm{DPPI}^{-/-}$mice (11-13). Levels of active proteinase-3 and tryptase are also reduced, indicating that DPPI regulates these peptidases as well $(11,12)$. Because the active form of many of these proteases is important for the host response (14-17), we used the cecal ligation and puncture (CLP) model of bacterial peritonitis to study how the absence of active DPPI and multiple serine proteases affects the host response to bacterial infection and sepsis.

\section{Methods}

Materials. All chemicals were from Sigma-Aldrich (St. Louis, Missouri, USA) unless otherwise noted.

Experimental animals. We used $\mathrm{DPPI}^{-/-}$mice backcrossed five generations into a C57BL/6 background, and $\mathrm{DPPI}^{+/+}$littermates as controls. C57BL/6 $\mathrm{Kit}^{W s b} / \mathrm{Kit}^{W s h}$ mice (18) were provided originally by Peter Besmer (Memorial Sloan-Kettering Institute, New 
York, New York, USA). C57BL/6 IL-6-/- mice were purchased from The Jackson Laboratory (Bar Harbor, Maine, USA) and then crossed with our $\mathrm{DPPI}^{-/-}$mice to generate $\mathrm{DPPI}^{-/-}$and $\mathrm{IL}^{-6^{-/}}$double-knockout mice (DPPI $I^{-/-} / \mathrm{IL}^{-6^{-/-}}$mice). All experimental procedures were performed in 8- to 12-week-old mice and were approved by the University of California San Francisco Committee on Animal Research.

Cecal ligation and puncture. A 1-cm midline incision was made in the abdominal wall of anesthetized mice and the cecum was identified. The distal $50 \%$ of exposed cecum was ligated with 3-0 silk suture and punctured with one pass of an 18-gauge needle. The cecum was replaced in the abdomen, the incision was closed with 3-0 suture, and the mouse recovered with a $0.5-\mathrm{ml}$ intraperitoneal injection of sterile $0.9 \% \mathrm{NaCl}$. Mice were monitored three times daily and survival was recorded. Moribund mice were euthanized by $\mathrm{CO}_{2}$ inhalation and cervical dislocation.

Measurement of markers of disease severity. Mice were weighed immediately prior to and 24 hours after CLP. Serum markers (alanine aminotransferase, blood urea nitrogen, and $\mathrm{CO}_{2}$ ) were quantified using a Synchron LX20 instrument (Beckman Coulter Inc., Fullerton, California, USA). Osmolality was measured using a microosmometer (Advanced Instruments Inc., Norwood, Massachusetts, USA) in serum harvested from blood obtained from mice 24 hours after CLP. As a measure of lung capillary leak, wet/dry lung weights were determined as previously described (19).

Peritoneal lavage. Anesthetized mice were euthanized by cervical dislocation and the abdominal skin was cleansed with $70 \%$ ethanol. Four milliliters of sterile $0.9 \% \mathrm{NaCl}$ was then instilled into the peritoneum. The abdomen was massaged gently for 1 minute and then opened with sterile scissors. Recovered peritoneal lavage fluid was centrifuged at 2,000 $\mathrm{g}$ for 4 minutes at $4^{\circ} \mathrm{C}$ and the supernatant was recovered for cytokine analysis. Cell pellets were resuspended in red blood cell lysis buffer (Sigma-Aldrich) for 15 minutes and recentrifuged, and the cell pellet was resuspended in PBS. Cell numbers were counted with a hemocytometer and cell differentials were determined on cytospun cells stained with Diff-Quik (American Scientific Products, McGaw Park, Illinois, USA).

Culture of mast cells from bone marrow. Mouse bone marrow mast cells (BMMCs) were cultured in WEHI3B-conditioned medium as described (20). Cells were used after 5 weeks in culture, at which time the cell populations consisted of more than $95 \%$ mast cells (identified by metachromatic granules in toluidine blue-stained cells).

Mast cell reconstitution of $\mathrm{Kit}^{W s h} / \mathrm{Kit}^{W s h}$ mice. We used C57BL/ $6 \mathrm{Kit}^{W s b} / \mathrm{Kit}^{W \mathrm{sh}}$ mast cell-deficient mice (21) for these studies because they have the same genetic background as the $\mathrm{DPPI}^{-/-}$mice, and at the age used in these studies, have no mast cells detectable by metachromatic staining in the spleen, stomach, small intestine, mesentery, or peritoneum. For reconstitu- tion experiments, $5 \times 10^{6} \mathrm{DPPI}^{+/+}$or $\mathrm{DPPI}^{-/-} \mathrm{BMMCs}$ suspended in $500 \mu \mathrm{l}$ of sterile PBS were injected into the peritoneum of 5-week-old $\mathrm{Kit}^{W s b} / \mathrm{Kit}^{W s b}$ mice. After allowing 5 weeks for mast cells to differentiate within the peritoneum $(3,22)$, the reconstituted mice were used in experiments. This method selectively reconstitutes peritoneal mast cells (PMCs) to similar levels in the mesentery or peritoneum $\left(4.0 \times 10^{5} \pm 0.5 \times 10^{5} \mathrm{vs}\right.$. $3.9 \times 10^{5} \pm 0.7 \times 10^{5}$ mast cells/peritoneum) using either $\mathrm{DPPI}^{+/+}$or $\mathrm{DPPI}^{-/-}$BMMCs.

Quantification of bacterial CFUs. Immediately after recovery, $10 \mu \mathrm{l}$ of peritoneal lavage fluid or blood was diluted serially in sterile $0.9 \% \mathrm{NaCl}$. Ten microliters of each dilution was aseptically plated and cultured on tryptose blood agar plates (Becton, Dickinson and Co., Sparks, Maryland, USA) at $37^{\circ} \mathrm{C}$. After 72 hours, the number of bacterial colonies was counted.

Cytokine analysis. Cytokine concentrations were measured in serum and peritoneal lavage fluid using ELISA kits for TNF- $\alpha$ (eBioscience, San Diego, California, USA), IL-1 $\beta$ (R\&D Systems Inc., Minneapolis, Minnesota, USA), IL-6 (eBioscience), and IL-10 (eBioscience) according to the manufacturer's protocol.

IL-6 hydrolysis. Five hundred ng of murine IL-6 (PeproTech Inc., Rocky Hill, New Jersey, USA) was incubated for three hours at $37^{\circ} \mathrm{C}$ in $10 \mu \mathrm{l}$ of PBS, in PBS containing a 1:200 molar ratio of mMCP-4 (23) or mMCP6 (23), and in PBS containing $4 \mathrm{mM}$ DTT and a 1:200 molar ratio of dog DPPI (24). Cleaved fragments were identified by SDS-PAGE.

Statistics. Survival curves were analyzed using the twotailed Fisher exact test. ANOVA followed by two-tailed $t$ testing was used to compare markers of organ dysfunction, bacterial CFUs, and mean cytokine concentrations. All calculations were performed using StatView 5.0.1 software (SAS Institute Inc., Cary, North Carolina, USA). Significance was assigned to $P$ values smaller than 0.05 .

\section{Results}

$D P I^{-/-}$mice are protected from CLP-induced death. Neutrophils and mast cells are required for efficient bacterial clearance and survival of CLP-mediated septic peritonitis $(3,25)$. Survival also depends on the presence of active granule-associated serine peptidases within these cells $(14,15,17)$. Because DPPI is required for activation of these enzymes (11-13), we expected to find decreased survival following CLP in $D P P I^{-/-}$mice. Surprisingly, we found that survival of $\mathrm{DPPI}^{-/-}$mice was more than twice that of $\mathrm{DPPI}^{+/+}$controls 8 days after CLP (51\% vs. $18 \%$; Figure 1). This survival advantage persists for up to 28 days after CLP as no late deaths were observed in mice from either group (data not shown). Thus, while the absence of active neutrophil elastase and cathepsin $G$ reduces survival following bacterial infection (14), survival is improved by a more severe disruption of serine protease activity (an absence of neutrophil elastase, cathepsin G, chymase, and granzymes A and B, and diminished proteinase- 3 and 


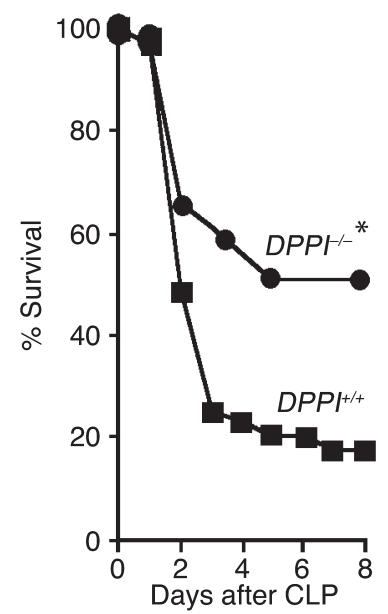

Figure 1

$D P I^{-/-}$mice are protected from death from septic peritonitis. $\mathrm{DPPl}^{+/+}$(39 mice) and $\mathrm{PPPI}^{-/-}$(41 mice) mice were subjected to CLP ( $50 \%$ ligation and single puncture with an 18-gauge needle), and survival was monitored for 8 days. Note the markedly improved survival of $D P P I^{-1-}$ mice. ${ }^{*} P=0.001$.

tryptase activity) in addition to DPPI. This suggests that the survival advantage caused by the genetic deletion of DPPI is not due to the absence of active neutrophil elastase or cathepsin G, and more remarkably, that it overcomes any harm caused by the absence of these neutral peptidases $(14,15)$.

Markers of organ dysfunction are similar in $\mathrm{DPPI}^{-1-}$ and $\mathrm{DPPI}^{+/+}$mice. We next measured markers of disease severity and organ damage in an attempt to explain the enhanced survival of $\mathrm{DPPI}^{-/-}$mice. Markers were measured shortly before the first deaths in this model to allow us to sample all mice (survivors and future nonsurvivors). At the 24-hour timepoint, $D P P I^{-/-}$mice had lost more weight than had $\mathrm{DPPI}^{+/+}$controls (Table 1 ). Markers of systemic acidosis $\left(\mathrm{CO}_{2}\right)$, hypovolemia (osmolality), and kidney, lung, and liver dysfunction (blood urea nitrogen, wet/dry lung weight, alanine aminotransferase) were similar in the two groups of mice at the 24-hour timepoint (Table 1). Similarly, the blood urea nitrogen and wet/dry lung weights were comparable in the two groups of mice 48 hours after CLP (data not shown). Finally, the number and types of inflammatory cells did not differ in the peritoneum 4 hours and 24 hours after CLP (Figure 2).

A complete physiologic explanation of death in the CLP model is lacking, but it appears that mice succumb from hypotension associated with septic shock (26). Our findings that markers of organ damage are similar in $\mathrm{DPPI}^{+/+}$and $\mathrm{DPPI}^{-/-}$mice demonstrate that the survival advantage of $D P P I^{-/-}$mice does not result from early protection of vital organs. Furthermore, the lack of a difference in wet/dry lung weights indicates that the survival advantage of $D P P I^{-/-}$mice does not stem from a reduction in the plasma leakage into the lung that was seen in septic neutrophil elastase-negative and cathepsin G-negative double-knockout mice (16). Our findings that the type and magnitude of cellular inflammation within the peritoneum are similar in $\mathrm{DPPI}^{+/+}$and $\mathrm{DPPI}^{-/-}$mice indicate that differences in cellular inflammation do not explain the survival advantage of $\mathrm{DPPI}^{-/-}$mice, and that DPPI and the serine proteases it activates are not required for migration of neutrophils from the intravascular compartment to the site of infection within the peritoneum. These findings are consistent with prior reports that DPPI, neutrophil elastase, and cathepsin $\mathrm{G}$ are not required for tissue penetration by neutrophils $(11,16)$.

Bacterial clearance is impaired in $\mathrm{DPPI}^{-/-}$mice. Because the primary objective of the host response to bacterial infection is removal of the organisms, we compared bacterial numbers in the peritoneum and blood of $\mathrm{DPPI}^{-/-}$ mice with those in $\mathrm{DPPI}^{+/+}$controls. Four hours after CLP, the number of peritoneal bacteria CFUs was similar in $\mathrm{DPPI}^{-/-}$and $\mathrm{DPPI}^{+/+}$mice (Figure 3a), indicating similar initial bacterial loads. However, 24 and 48 hours after CLP, $D P P I^{-/}$mice had far more bacteria in the peritoneum (Figure 3, b and d) and blood (Figure 3c) than $\operatorname{did} \mathrm{DPPI}^{+/+}$mice, demonstrating that $\mathrm{DPPI}^{-/-}$mice have impaired bacterial control and clearance.

Four weeks after CLP, all surviving mice had developed abscesses in the region of the cecum. Yet despite this mechanical control of the infection, $\mathrm{DPPI}^{-/-}$mice continued to have more bacteria in the peritoneum and blood than did $\mathrm{DPPI}^{+/+}$mice (Figure 3, e and f), albeit at markedly lower levels than at 48 hours after CLP. This indicates that the impairment in bacterial clearance persists throughout the course of the infection. Because survival from sepsis depends, in part, on efficient removal of pathogenic bacteria, we were surprised by our findings that $\mathrm{DPPI}^{-/-}$mice have an impairment in bacterial control and clearance. These findings indicate that the improved survival of $\mathrm{DPPI}^{-/-}$mice is independent of bacterial clearance and that the survival advantage is the consequence of altered inflammatory response to the infection.

To determine whether mast cell DPPI plays a role in bacterial clearance, we selectively reconstituted the peritoneums of mast cell-deficient $\mathrm{Kit}^{W \mathrm{Ws}} / \mathrm{Kit}^{W \mathrm{Ws}}$ mice with $D P P I^{-/-}$-derived BMMCs, i.e., PMC-specific $D P P I^{-/-}$ mice, and found that PMC-DPPI ${ }^{-/}$mice have numbers of intraperitoneal bacteria similar to $\mathrm{DPPI}^{+/+}$mice 24 hours after CLP (Figure $3 \mathrm{~b}$ ). This finding clearly shows

\section{Table 1}

Markers of disease severity 24 hours after cecal ligation and puncture

$\begin{array}{lccc} & & & \\ \text { Marker } & D P P I^{+/+} & D P P I^{-/-} & P \text { value } \\ \% \text { wt loss } & 2.6 \% \pm 1.7 \% & 6.6 \% \pm 1.9 \% & 0.007 \\ \mathrm{ALT} & 150 \pm 35 & 215 \pm 110 & 0.090 \\ \mathrm{BUN} & 55 \pm 25 & 51 \pm 30 & 0.806 \\ \mathrm{CO}_{2} & 16.4 \pm 2.9 & 15.8 \pm 3.0 & 0.862 \\ \text { Osmolality } & 348 \pm 10 & 355 \pm 12 & 0.820 \\ \text { Wet/dry lung wt } & 3.4 \pm 0.2 & 3.4 \pm 0.2 & 0.958\end{array}$

ALT, alanine aminotransferase; BUN, blood urea nitrogen. $n=5-8$ mice per group. 

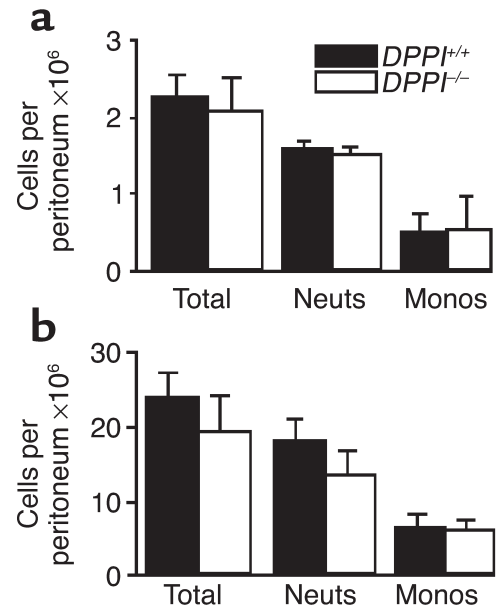

Figure 2

Intraperitoneal inflammatory cell recruitment after CLP is similar in $\mathrm{DPPl}^{+/+}$and $\mathrm{DPPI} I^{-/-}$mice. $\mathrm{DPPl}{ }^{+/+}$and $\mathrm{DPPI}^{-/-}$mice (7-9 mice per group) were euthanized 4 hours (a) and 24 hours (b) after CLP, and intraperitoneal inflammatory cells were recovered by peritoneal lavage. Total cells were counted using a hemocytometer and the cell differentials were determined on cytospun cells stained with DiffQuik. Monos, mononuclear cells; neuts, neutrophils.

that the lack of mast cell DPPI is not responsible for the excessive bacteria in $\mathrm{DPPI}^{-/-}$mice. $\mathrm{PMC}-\mathrm{DPPI} \mathrm{I}^{-/-}$mice lack DPPI only within their PMCs. All other cells have normal amounts of DPPI and the proteases it activates. Thus, impaired bacterial clearance more likely results from an absence of active neutrophil elastase and cathepsin G. These proteases play essential roles in bacterial killing and clearance $(14,15)$, as shown in neutrophil elastase- and cathepsin G-negative mice, in which bacterial clearance is impaired in various mouse models of bacterial infection.

Intraperitoneal and systemic expression of $I L-6$ is altered in $\mathrm{DPPI}^{-/-}$mice. Prior work has shown that DPPI influences the level of inflammatory cytokines in the subcutaneous air pouches of mice injected with zymosan
(11). To determine whether altered cytokine expression is responsible for the improved survival of $\mathrm{DPPI}^{-/-}$mice, we next compared local levels of cytokines at the site of infection within the peritoneum and systemic levels in the serum of $\mathrm{DPPI}^{-{ }^{--}}$and $\mathrm{DPPI}^{+/+}$mice. At multiple times between 2 hours and 24 hours after CLP, the intraperitoneal levels of TNF- $\alpha$, IL- $1 \beta$, and IL-10 were comparable in $\mathrm{DPPI}^{-/-}$and $\mathrm{DPPI}^{+/+}$mice (Figure 4, a, b, and d). In contrast, intraperitoneal IL-6 was significantly higher in $\mathrm{DPPI}^{-/-}$mice than in $\mathrm{DPPI}^{+/+}$mice 2 hours and 6 hours after CLP (Figure 4c). Similarly, IL- 6 serum levels were markedly higher in $\mathrm{DPPI}^{-/-}$mice 24 hours after CLP (Figure 4e). Interestingly, relative levels of IL- 6 mRNA (quantified by real-time PCR) were slightly (1.8-fold) higher in peritoneal lavage cells obtained from $D P P I^{--}$mice than in those obtained from $\mathrm{DPPI}^{+/+}$mice 2 hours after CLP (data not shown). By 6 hours this small difference disappeared. These results suggest that the elevated levels of IL- 6 do not result from altered $I L-6$ gene expression in $D P \mathrm{PI}^{-1-}$ mice.

To test whether elevated levels of IL- 6 mediate the survival advantage in $\mathrm{DPPI}^{-/-}$mice, we compared survival of $D P P I^{-/-}$mice to that of mice lacking expression of both DPPI and IL-6 (DPPI-/-/IL-6 $6^{-/-}$mice) and found that survival of $D P P I^{-/-} / I L-6^{-/}$mice is significantly worse than survival of $\mathrm{DPPI}^{-/-}$mice and identical to that of $\mathrm{DPPI}^{+/+}$and $\mathrm{IL}^{-6^{-/-}}$mice 4 days after CLP (Figure 5 ). This indicates that the survival advantage in $D P P I^{-/-}$ mice depends on IL- 6 expression.

The marked elevation in IL- 6 levels and the observation that removal of IL- 6 expression eliminates the survival advantage in $\mathrm{DPPI}^{-/-}$mice strongly suggests that the survival benefit in $D P P I^{-/-}$mice is dependent on IL-6. This is consistent with prior studies showing that administration of recombinant IL- 6 protects mice from death following LPS-mediated (27) or staphylococcal enterotoxin-mediated septic shock (28), and that $I L-6^{-/-}$mice have greater mortality when infected with Escherichia coli (25) or Streptococcus pneumonia (29), and following injection with endotoxin (30). Thus,

\section{Figure 3}

Bacterial clearance is impaired in $\mathrm{DPPI}^{-1-}$ mice. Dilutions of peritoneal lavage fluid obtained from $\mathrm{DPPI}^{+/+}$, $\mathrm{DPPI}^{-/-}$, and $K i t^{W h} / K i t^{W s h}$ mice selectively reconstituted with $D P P I^{-1-}$ BMMCs (PMC-DPPI-/- mice) 4 hours (a), 24 hours (b), 48 hours (d), or 4 weeks (e) after CLP, and blood obtained 24 hours (c) or 4 weeks (f) after CLP were cultured on tryptose blood agar plates, and the number of bacterial colonies was counted. $n=6-9$ mice per group. ${ }^{*} P<0.05$.
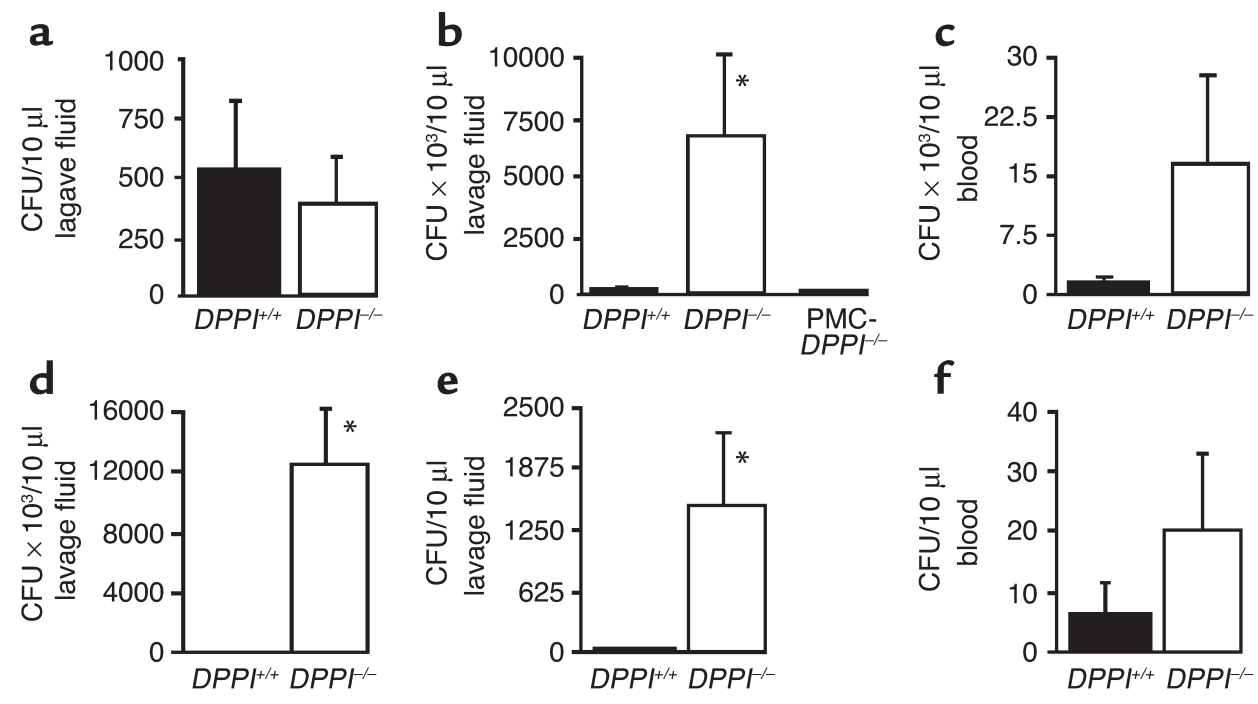

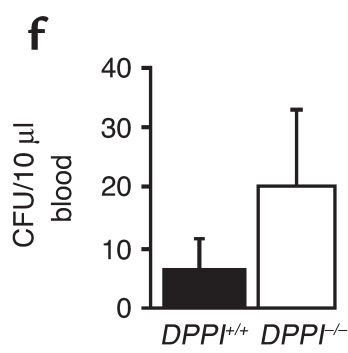




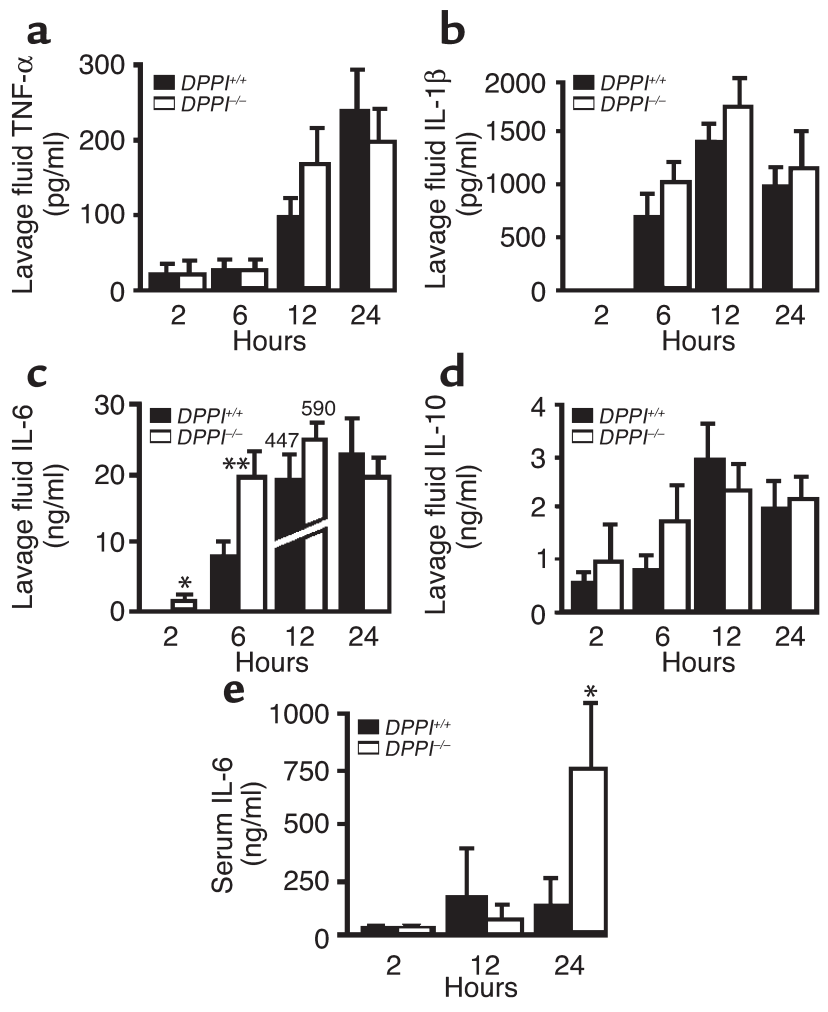

while it has been observed previously that IL-6 is important for host survival of sepsis, our discovery of higher IL-6 levels in $\mathrm{DPPI}^{-/-}$mice suggests a novel role for DPPI in regulating IL-6 and survival in septic mice.

A lack of mast cell DPPI accounts for the survival advantage of septic DPPI ${ }^{-/}$mice. Because DPPI is expressed in several types of innate immune cells, we next tested whether the selective absence of DPPI in one of these cells, the mast cell, contributes to the survival benefit. To accomplish this, we compared the survival of PMC-DPPI-/mice to that of mast cell-deficient $K i t^{W s b} / K i t^{W s b}$ mice and $\mathrm{Kit}^{W s b} / \mathrm{Kit}^{W s b}$ mice selectively reconstituted with $D P P I^{+/+}$ BMMCs (PMC-DPPI ${ }^{+/+}$mice). We found that survival in $\mathrm{Kit}^{W s b} / \mathrm{Kit}^{W s b}$ mice was worse than in PMC-DPPI ${ }^{+/+}$mice (10\% vs. $26 \%$, Figure $6 a)$, and that survival in PMC$\mathrm{DPPI}^{-/-}$mice was markedly better than in PMC-DPPI ${ }^{+/+}$ mice ( $47.5 \%$ vs. $26 \%$ ) and was nearly identical ( $47.5 \%$ vs. $50 \%$ ) to that in $\mathrm{DPPI}^{-/-}$mice (Figure 6a and Figure 1).

The observation that survival in mast cell-deficient $\mathrm{Kit}^{\mathbb{W s b}} / \mathrm{Kit}^{\mathbb{W} s \mathrm{~s}}$ mice is worse than in $\mathrm{DPPI}^{+/+}$mice and PMC$\mathrm{DPPI}^{+/+}$mice is consistent with prior studies reporting that survival of $\mathrm{Kit}^{\mathbb{W}} / \mathrm{Kit}^{\mathbb{W v}}$ mast cell-deficient mice is worse than in mast cell-sufficient littermate controls and that intraperitoneal reconstitution of $\mathrm{Kit}^{\mathbb{W}} / \mathrm{Kit}^{\mathbb{W} v}$ mice with mast cells restores survival to near that of controls $(3,31)$. More importantly, by showing that survival in PMC-DPPI-/- mice is significantly better than in PMC$\mathrm{DPPI}^{+/+}$mice and almost identical to that of $\mathrm{DPPI}^{-/-}$ mice, we prove that the $D P P I^{-/}$survival advantage is due to the absence of mast cell DPPI. This observation differs from previous studies reporting a protective role for mast cells in septic peritonitis $(3,31)$, and shows for the

\section{Figure 4}

IL- 6 levels are elevated in the peritoneum and serum of $D P \mathrm{PI}^{-/-}$mice. Concentrations of TNF- $\alpha$ (a), IL-1 $\beta$ (b), IL- 6 (c), and IL-10 (d) were measured by ELISA in peritoneal lavage fluid (a-d) and IL- 6 in serum (e) that was obtained at various time points after CLP. $n=7-9$ mice per timepoint. ${ }^{*} P<0.05,{ }^{*} P=0.006$.

first time that at least one mast cell product (DPPI) has the opposite effect and enhances mortality from sepsis.

To establish that mast cell DPPI modulates levels of peritoneal IL-6, we first measured IL-6 levels in the peritoneums of PMC-DPPI-/- and PMC-DPPI ${ }^{+/+}$mice and found that they were higher in the peritoneums of mice lacking only mast cell DPPI (PMC-DPPI ${ }^{-/}$) 2 hours after CLP (Figure 6b). This demonstrates that mast cell DPPI regulates peritoneal IL-6 levels following CLP. Finally, we tested whether the elevated IL-6 levels in $D P I^{-1-}$ mice could result from diminished activity of IL-6-destroying mast cell proteases. Following incubation of murine IL- 6 with chymase (mMCP-4), DPPI, or tryptase (mMCP-6), we found that tryptase (Figure 6c, lane 4) selectively cleaves IL-6, as demonstrated by the appearance of a new 23-kDa band. Chymase (Figure 6c, lane 2) and DPPI (Figure 6c, lane 3) also hydrolyze IL-6, but to a lesser degree. These results show that higher IL-6 levels in septic $\mathrm{DPPI}^{-/-}$mice are due to reduced quantities of active mast cell proteases, especially tryptase, expressed in these animals (12).

\section{Discussion}

In summary, these studies show that mast cell DPPI critically regulates survival from sepsis. Our discovery that $D P I^{-/-}$mice are protected from death due to sepsis pre-

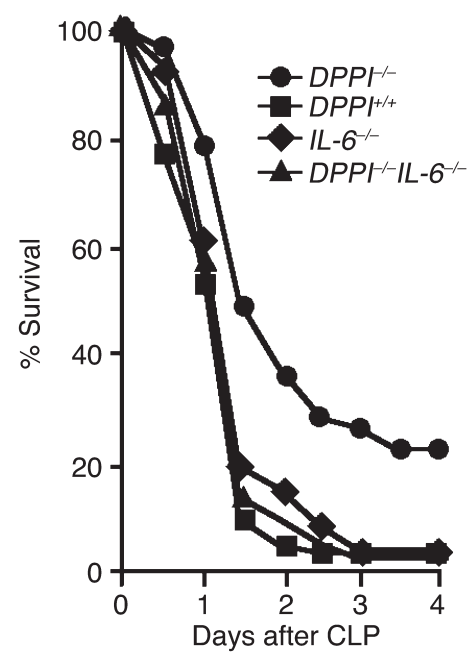

\section{Figure 5}

Removal of IL- 6 expression in $D P \mathrm{PI}^{-/-}$mice abolishes the survival advantage. DPPI-/- mice $(n=46), D P P I^{-/-} / \mathrm{IL}^{-6^{-/-}}$double-knockout mice $(n=45), D P P I^{+/+}$mice $(n=40)$, and $I L-6^{-/-}$mice $(n=27)$ were subjected to CLP, and survival was monitored for 5 days. Note that survival of $D P \mathrm{Pl}^{-/-} / \mathrm{IL-} 6^{-/-}$mice is significantly worse than that of $D P P I^{-/-}$mice. ${ }^{*} P=0.001$ for $D P P I^{-/-}$vs. $D P P I^{-/-} / I L-6^{-/-}$mice, and $P=0.008$ for $\mathrm{DPPI}^{-/-}$vs. $D \mathrm{PPI}^{+/+}$mice. 


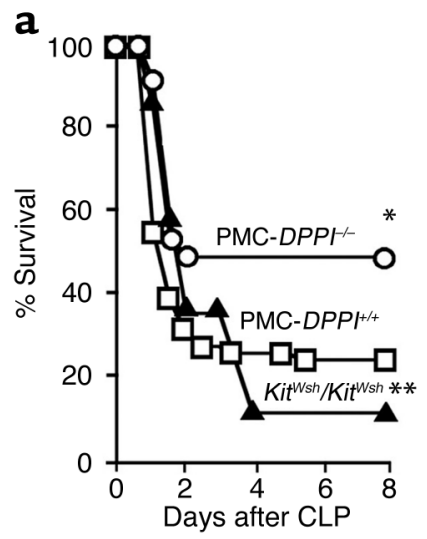

b

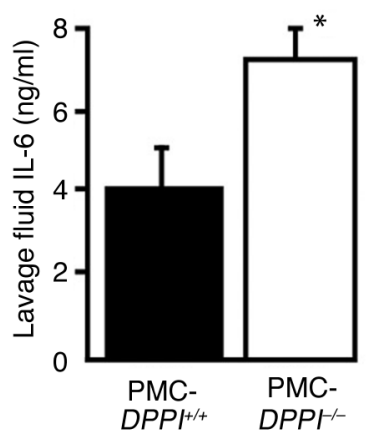

C

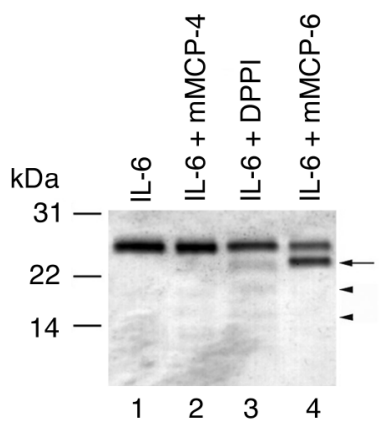

Figure 6

Selective absence of mast cell DPPI improves survival from sepsis. (a) Survival of mast cell-deficient Kit ${ }^{W s h} / K_{i t}{ }^{W s h}$ mice selectively reconsti-

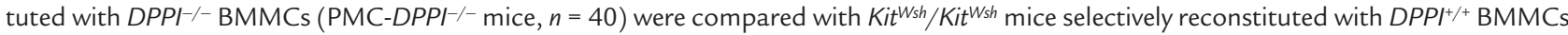
(PMC-DPPI+/+ mice, $n=65)$, and with $\mathrm{Kit}^{\text {Wh }} / \mathrm{Kit}^{\text {wsh }}(n=20)$ mice for 8 days following CLP. Note that survival of mice lacking DPPI solely in mast cells (PMC-DPPI-/- mice) is significantly better than that of PMC-DPPI ${ }^{+/+}$mice. ${ }^{*} P=0.026$ for PMC-DPPI-/- vs. PMC-DPPI ${ }^{+/+}$mice. ${ }^{*} P=0.22$ for $K_{i t}{ }^{W h} / K_{i t}{ }^{W s h}$ vs. PMC-DPPI $+/+$ mice. (b) IL-6 levels are higher in the peritoneal lavage of PMC-DPPI-/- mice. Concentrations of IL- 6 were measured by ELISA in lavage fluid obtained from PMC-DPPI ${ }^{+/}$and PMC-DPPI-/- mice 2 hours after CLP. ${ }^{*} P=0.05$ for PMC$\mathrm{DPPI}^{-/-}$mice vs. PMC-DPPI+/+ mice. (c) Mast cell proteases cleave IL-6. IL-6 was incubated alone (lane 1) or with mMCP-4 (lane 2), DPPI (lane 3), or mMCP-6 (lane 4) for 3 hours at $37^{\circ} \mathrm{C}$. Note the appearance of a new 23-kDa band in the mMCP-6-treated sample (black arrow), and new, faint bands in the DPPI-treated sample (arrowheads).

dicts that mast cell DPPI enhances the likelihood of death from sepsis in WT mice, notwithstanding the net protective role of mast cells suggested here and in prior studies in mast cell-deficient mice. Our findings are significant because they are the first to show that mast cell products can contribute to death of the septic host. Furthermore, because mast cells are one of the first cells to recognize the presence of an infection (32), mast cell DPPI may determine host survival at the time of mast cell activation shortly after the onset of infection.

Two key determinants of sepsis survival are bacterial clearance and the inflammatory response to the infection. Neutrophil elastase and cathepsin $\mathrm{G}$ are inactive in $\mathrm{DPPI}^{-/-}$mice (11). These proteases play essential roles in the killing and clearance of bacteria. This has been demonstrated in studies on elastase- and cathep$\sin$ G-negative mice, which have higher bacterial loads and mortality when infected with gram-negative or gram-positive bacteria, respectively $(14,15)$. The absence of active forms of these enzymes in $\mathrm{DPPI}^{-/-}$ mice impairs bacterial clearance and substantially raises the bacterial load 24 hours after CLP. Remarkably, $D P I^{-/-}$mice are far more likely to survive despite this defect in bacterial clearance. Thus, enhancing bacterial clearance is not required for improving sepsis survival in this model. However, modifying the timing of release, cellular expression, and levels of inflammatory cytokines is also important. Furthermore, if the survival advantage produced by altered cytokine expression is sufficiently great, it can even overcome high bacterial loads, as is the case for $\mathrm{DPPI}^{-/-}$mice.

IL-6 is a pleiotropic cytokine whose actions can be either pro- or anti-inflammatory $(30,33,34)$. Whether its role in sepsis, on balance, is protective or harmful has not yet been fully resolved. This conflict is exemplified by studies reporting that exogenously administered IL-6 improves survival in mouse models of sepsis and that mortality of $\mathrm{IL}^{-6^{-/}}$mice is high following bacterial infections compared with that of WT mice (25, 27-29). Conversely, treatment of mice with an IL-6blocking antibody improves survival from septic peritonitis, apparently by reducing expression of C5a receptor (35). However, this survival benefit occurs only in mice pretreated with the IL-6-blocking antibody and not in mice treated after initiation of peritonitis. Our finding that IL-6 levels are high in $\mathrm{DPPI}^{-/-}$mice and that deletion of IL- 6 expression eliminates the survival advantage in $\mathrm{DPPI}^{-/-}$mice is consistent with a protective effect of IL- 6 on the host from death during sepsis. How IL-6 protects the host is unknown, but our observations, combined with those of past studies, suggest that its actions may be related to the timing and magnitude of IL-6 peaks in specific tissue compartments.

The studies using PMC-DPPI-/- mice show that the survival advantage in $\mathrm{PPPI}^{-/-}$mice is due to the absence of mast cell DPPI. Prior studies have reported that mast cells protect mice from CLP-mediated sepsis (3). Our results show for the first time that in addition to providing protection, mast cells also contribute to sepsis mortality by producing a product (DPPI) that harms the host. The observations that the survival advantage in $\mathrm{DPPI}^{-/-}$mice is associated with elevated IL-6 levels and that the mast cell tryptase mMCP- 6 hydrolyzes IL-6 raise the intriguing possibility that mast cell DPPI controls levels of IL- 6 by regulating production of tryptase and possibly other IL-6-destroying peptidases. A direct effect of DPPI on IL-6 is unlikely given the observed slow rate of hydrolysis by DPPI in vitro. The 
hypothesized importance of mMCP-6 (and possibly other tryptases) is attractive not only because of this study's observation that it hydrolyzes IL- 6 in vitro but because levels of active mMCP- 6 are sharply reduced in mast cells of $\mathrm{DPPI}^{-/-}$mice (12). Thus, we propose that the following sequence of events occurs in the setting of an infection: Bacteria activate mast cells, stimulating secretion of cytokines such as TNF- $\alpha$ and IL- $6(5,36)$. Secreted TNF- $\alpha$ aids in recruiting neutrophils to the site of infection. At the same time, activated mast cells release tryptases, which reduce IL-6 levels via proteolytic inactivation. Because IL- 6 is beneficial at certain times during the septic response, inactivation by tryptase is harmful, and if levels drop below a critical threshold, the animal dies. For the individual host, the net harm or benefit of mast cell products depends on the balance of these opposing processes. Whether similar processes occur for other mediators untested in this model is unknown.

In summary, the data presented here show that mast cell DPPI is a key regulator of survival from septic peritonitis. These findings change the prevailing view that mast cells protect the host and demonstrate that mast cells can mediate septic death. It is intriguing to speculate that by inhibiting the activity of mast cell DPPI we may be able to improve outcomes from sepsis.

\section{Acknowledgments}

The authors thank Timothy Ley for providing the $\mathrm{DPPI}^{-/-}$mice; Michael Matthay, Wilfred Raymond, and Amy Sehnert for helpful discussions; Xiaohui Fang for assisting in measurements of wet/dry lung weights; and Andrew McKinstry for his excellent technical support. This work was supported in part by the American Lung Association of California (P.J. Wolters), a Biomedical Science Award from the Arthritis Foundation (C.T.N. Pham), and grants HL-04055 (P.J. Wolters), AI-49261 (C.T.N. Pham), and HL-024136 (G.H. Caughey) from the NIH.

1. Cohen, J. 2002. The immunopathogenesis of sepsis. Nature. 420:885-891.

2. Janeway, C.A., Jr., and Medzhitov, R. 2002. Innate immune recognition. Annu. Rev. Immunol. 20:197-216.

3. Echtenacher, B., Mannel, D.N., and Hultner, L. 1996. Critical protective role of mast cells in a model of acute septic peritonitis. Nature. 381:75-77.

4. Malaviya, R., Ikeda, T., Ross, E., and Abraham, S.N. 1996. Mast cell modulation of neutrophil influx and bacterial clearance at sites of infection through TNF-alpha. Nature. 381:77-80.

5. Supajatura, V., et al. 2002. Differential responses of mast cell Toll-like receptors 2 and 4 in allergy and innate immunity. J. Clin. Invest. 109:1351-1359. doi:10.1172/JCI200214704.

6. Malaviya, R., Gao, Z., Thankavel, K., van der Merwe, P.A., and Abraham, S.N. 1999. The mast cell tumor necrosis factor alpha response to FimHexpressing Escherichia coli is mediated by the glycosylphosphatidylinositol-anchored molecule CD48. Proc. Natl. Acad. Sci. U. S. A. 96:8110-8115.

7. Gommerman, J.L., et al. 2000. A role for CD21/CD35 and CD19 in responses to acute septic peritonitis: a potential mechanism for mast cell activation. J. Immunol. 165:6915-6921.

8. Belaaouaj, A., Kim, K.S., and Shapiro, S.D. 2000. Degradation of outer membrane protein A in Escherichia coli killing by neutrophil elastase. Science. 289:1185-1188.

9. Weinrauch, Y., Drujan, D., Shapiro, S.D., Weiss, J., and Zychlinsky, A.
2002. Neutrophil elastase targets virulence factors of enterobacteria. Nature. 417:91-94.

10. McGuire, M.J., Lipsky, P.E., and Thiele, D.L. 1992. Purification and characterization of dipeptidyl peptidase I from human spleen. Arch. Biochem. Biophys. 295:280-288.

11. Adkison, A.M., Raptis, S.Z., Kelley, D.G., and Pham, C.T. 2002. Dipeptidyl peptidase I activates neutrophil-derived serine proteases and regulates the development of acute experimental arthritis. J. Clin. Invest. 109:363-371. doi:10.1172/JCI200213462.

12. Wolters, P.J., Pham, C.T., Muilenburg, D.J., Ley, T.J., and Caughey, G.H. 2001. Dipeptidyl peptidase I is essential for activation of mast cell chymases, but not tryptases, in mice. J. Biol. Chem. 276:18551-18556.

13. Pham, C.T., and Ley, T.J. 1999. Dipeptidyl peptidase I is required for the processing and activation of granzymes A and B in vivo. Proc. Natl. Acad. Sci. U. S. A. 96:8627-8632.

14. Belaaouaj, A., et al. 1998. Mice lacking neutrophil elastase reveal impaired host defense against gram negative bacterial sepsis. Nat. Med. 4:615-618.

15. Reeves, E.P., et al. 2002. Killing activity of neutrophils is mediated through activation of proteases by K+ flux. Nature. 416:291-297.

16. Tkalcevic, J., et al. 2000. Impaired immunity and enhanced resistance to endotoxin in the absence of neutrophil elastase and cathepsin G. Immunity. 12:201-210.

17. Huang, C., et al. 2001. Evaluation of the substrate specificity of human mast cell tryptase beta I and demonstration of its importance in bacterial infections of the lung. J. Biol. Chem. 276:26276-26284.

18. Duttlinger, R., et al. 1995. The Wsh and Ph mutations affect the c-kit expression profile: c-kit misexpression in embryogenesis impairs melanogenesis in Wsh and $\mathrm{Ph}$ mutant mice. Proc. Natl. Acad. Sci. U. S. A. 92:3754-3758.

19. Jayr, C., et al. 1994. Alveolar liquid and protein clearance in anesthetized ventilated rats. J. Appl. Physiol. 76:2636-2642.

20. Razin, E., et al. 1984. Interleukin 3: a differentiation and growth factor for the mouse mast cell that contains chondroitin sulfate E proteoglycan. J. Immunol. 132:1479-1486.

21. Tono, T., et al. 1992. c-kit Gene was not transcribed in cultured mast cells of mast cell-deficient Wsh/Wsh mice that have a normal number of erythrocytes and a normal c-kit coding region. Blood. 80:1448-1453.

22. Nakano, T., et al. 1985. Fate of bone marrow-derived cultured mast cells after intracutaneous, intraperitoneal, and intravenous transfer into genetically mast cell-deficient W/Wv mice. Evidence that cultured mast cells can give rise to both connective tissue type and mucosal mast cells. J. Exp. Med. 162:1025-1043.

23. Coussens, L.M., et al. 1999. Inflammatory mast cells up-regulate angiogenesis during squamous epithelial carcinogenesis. Genes Dev. 13:1382-1397.

24. Wolters, P.J., Raymond, W.W., Blount, J.L., and Caughey, G.H. 1998. Regulated expression, processing, and secretion of dog mast cell dipeptidyl peptidase I. J. Biol. Chem. 273:15514-15520.

25. Dalrymple, S.A., et al. 1996. Interleukin-6 is required for a protective immune response to systemic Escherichia coli infection. Infect. Immun. 64:3231-3235.

26. Hollenberg, S.M., et al. 2001. Characterization of a hyperdynamic murine model of resuscitated sepsis using echocardiography. Am. J. Respir. Crit. Care Med. 164:891-895.

27. Barton, B.E., and Jackson, J.V. 1993. Protective role of interleukin 6 in the lipopolysaccharide-galactosamine septic shock model. Infect. Immun. 61:1496-1499.

28. Barton, B.E., Shortall, J., and Jackson, J.V. 1996. Interleukins 6 and 11 protect mice from mortality in a staphylococcal enterotoxin-induced toxic shock model. Infect. Immun. 64:714-718.

29. van der Poll, T., et al. 1997. Interleukin-6 gene-deficient mice show impaired defense against pneumococcal pneumonia. J. Infect. Dis. 176:439-444.

30. Xing, Z., et al. 1998. IL-6 is an antiinflammatory cytokine required for controlling local or systemic acute inflammatory responses. J. Clin. Invest. 101:311-320

31. Maurer, M., et al. 1998. The c-kit ligand, stem cell factor, can enhance innate immunity through effects on mast cells. J. Exp. Med. 188:2343-2348.

32. Galli, S.J., Maurer, M., and Lantz, C.S. 1999. Mast cells as sentinels of innate immunity. Curr. Opin. Immunol. 11:53-59.

33. Ohshima, S., et al. 1998. Interleukin 6 plays a key role in the development of antigen-induced arthritis. Proc. Natl. Acad. Sci. U. S. A. 95:8222-8226.

34. Fattori, E., et al. 1994. Defective inflammatory response in interleukin 6-deficient mice. J. Exp. Med. 180:1243-1250.

35. Riedemann, N.C., et al. 2003. Protective effects of IL-6 blockade in sepsis are linked to reduced c5a receptor expression. J. Immunol. 170:503-507.

36. McCurdy, J.D., Lin, T.J., and Marshall, J.S. 2001. Toll-like receptor 4mediated activation of murine mast cells. J. Lenkoc. Biol. 70:977-984. 